\title{
One-Step Electro-Precipitation of Nanocellulose Hydrogels on Conducting Substrates and Its Possible Applications: Coatings, Composites, and Energy Devices
}

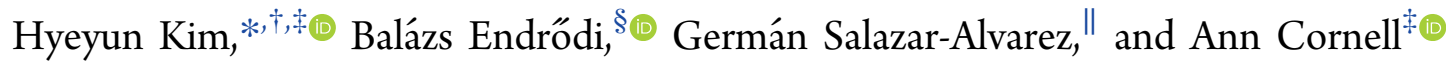 \\ ${ }^{\dagger}$ Wallenberg Wood Science Centre, Royal Institute of Technology, KTH Royal Institute of Technology, Teknikringen 56-58, SE-100 \\ 44 Stockholm, Sweden \\ ${ }^{\ddagger}$ Department of Chemical Engineering, KTH Royal Institute of Technology, Teknikringen 42, SE-100 44 Stockholm, Sweden \\ ${ }^{\S}$ Department of Physical Chemistry and Materials Science, University of Szeged, Rerrich Béla Square 1, H-6720 Szeged, Hungary \\ "Department of Materials and Environmental Chemistry, Stockholm University, Svante Arrhenius väg 16 C, SE-106 91 Stockholm, \\ Sweden
}

\section{Supporting Information}

ABSTRACT: TEMPO-oxidized cellulose nanofibrils (TOCN) are $\mathrm{pH}$-responsive biopolymers which undergo sol-gel transition at acidic conditions $(\mathrm{pH}<4)$ due to charge neutralization. Electronically conducting materials can be coated by such gels during aqueous electrolysis, when an electrochemical reaction generates a local $\mathrm{pH}$ decrease at the electrode surface. In this work, electro-precipitation of different TOCN gels has been performed on oxygen evolving anodes. We demonstrate that TOCN hydrogels can be electrochemically coated on the surface of any conductive material with even complex 3D shape. Further, not only TOCN but also micro- or nanosized particles containing TOCN composites can be coated on the electrode surface, and coatings containing multiple layers of different composites can be also produced. We demonstrate that this simple and facile electrocoating technique can be subject to various applications, such as coatings making electrodes selective for the hydrogen evolution reaction, as well as a new eco-friendly aqueous-based synthesis of Li-ion battery electrodes.

KEYWORDS: Cellulose, TEMPO-oxidized cellulose, Electro-precipitation, pH-responsive hydrogel, Stimuli-responsive hydrogel

\section{INTRODUCTION}

Electro-precipitation is a facile technique to produce materials and to coat a target substrate in response to electrical signals. Stimuli responsive biopolymers, e.g., chitosan and alginate, can form hydrogels, well-suited for applications in biomedical or tissue engineering and a variety of versatile soft matter applications. ${ }^{1-7}$ The most widely used mechanisms of electroprecipitation of such biopolymer hydrogels are reactions induced by (i) metal-ions coordination ${ }^{1-3,7}$ or (ii) $\mathrm{pH}$ dependent sol-gel transition. ${ }^{5,6,8}$

Electrochemical water splitting can be used to generate $\mathrm{pH}$ gradients at an electrode surface, which can result in the consequent sol-gel transition. In a water splitting electrochemical cell, the reaction on the anode is oxygen evolution (OER), resulting in proton and oxygen gas formation and hence a local $\mathrm{pH}$ decrease, $\left(2 \mathrm{H}_{2} \mathrm{O} \rightarrow \mathrm{O}_{2}(\mathrm{~g})+4 \mathrm{H}^{+}(\mathrm{aq})+4 \mathrm{e}^{-}\right)$. On the cathode, hydrogen gas and hydroxide ions are produced (HER), leading to a local $\mathrm{pH}$ increase, $\left(2 \mathrm{H}_{2} \mathrm{O}+2 \mathrm{e}^{-} \rightarrow \mathrm{H}_{2}(\mathrm{~g})+2 \mathrm{OH}^{-}\right)$.

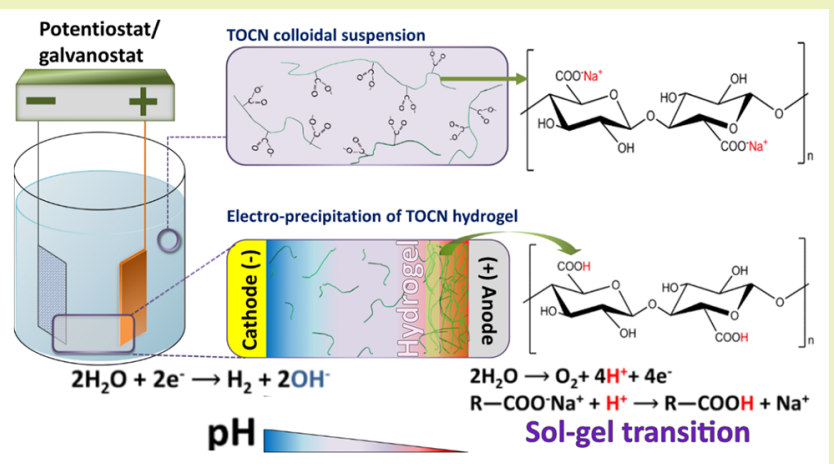

Such localized $\mathrm{pH}$ changes can result in sol-gel transition of $\mathrm{pH}$ responsive biopolymers. Taking the example of chitosan, hydroxide ions neutralize the polymer chain, leading to its gelation at the cathode surface (chitosan $-\mathrm{NH}_{3}{ }^{+}+\mathrm{OH}^{-} \rightarrow$ chitosan $\left.-\mathrm{NH}_{2}+\mathrm{H}_{2} \mathrm{O}\right){ }^{8}$ As another example, the localized $\mathrm{pH}$ decrease at the anode can neutralize the charge of sodium alginate substituting sodium to proton $\left(\mathrm{COO}^{-} \mathrm{Na}^{+}+\mathrm{H}^{+} \rightarrow\right.$ $\left.\mathrm{COOH}+\mathrm{Na}^{+}\right)$, causing the precipitation of adjacent polyelectrolytes on the anode. ${ }^{5}$ Since the electrochemical reaction induced precipitations take place in the diffusion layer of an electrode, it is advantageous that the growth of the hydrogel can be effectively controlled by varying the experimental conditions, such as the applied current or voltage.

Cellulose and its derivatives have been reported as promising hydrogel materials for versatile applications, such as biomedical

Received: July 19, 2019

Revised: October 15, 2019

Published: November 19, 2019 
engineering, drug delivery, tissue engineering and adsorbents. $^{9-12}$ TEMPO (2,2,6,6-tetramethylpiperidine-1-oxyl)-mediated oxidation of cellulose nanofibers (TOCN) leads to the substitution of hydroxyl groups at C6 positions of the glucose units to sodium carboxylate. These extra negative charges introduced on the cellulose chain cause strong electrostatic repulsion between the fibrils. Combined with consecutive mechanical treatment, individualized nanosized TOCN can be dispersed in aqueous solutions with high colloidal stability. Sodium counterions of C6 carboxylic groups stabilize the TOCN dispersion, but its colloidal properties, and most importantly the stability, change with the solution $\mathrm{pH}$. In acidic conditions, where $\mathrm{pH}$ is below the $\mathrm{p} K_{\mathrm{a}}$ of TOCN $(\sim 4.8)$, sodium counterions are dissociated from the sodium carboxylate groups and substituted by protons, forming carboxylic acid groups. Such charge neutralization significantly reduces the repulsion forces between nanofibrils, which loses its colloidal stability, resulting in the aggregation of TOCN..$^{10,13-16}$ In the same manner with sodium alginate, we show that a localized $\mathrm{pH}$ decrease at an oxygen evolving anode can fabricate a TOCN based hydrogel at the anode surface. The mechanism of $\mathrm{pH}$ responsive sol-gel transition of TOCN induced by water oxidation is described in Figure 1.

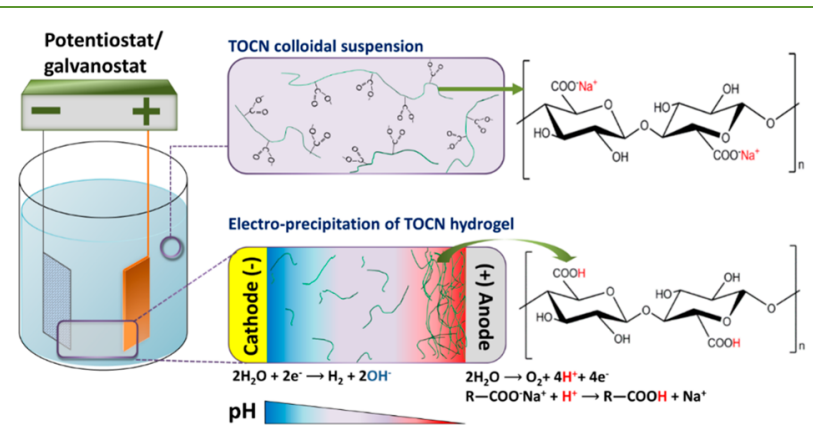

Figure 1. Schematic illustrations of the primary mechanism of $\mathrm{pH}$ responsive sol-gel transition of TOCN induced by water oxidation at an anode

As a further benefit of electro-precipitation, nanostructured composite materials can be easily fabricated. ${ }^{2,5,7,17}$ Nanoscale particles dispersed in the coating dispersion with stimuli responsive biopolymers can get immobilized on the conductive substrates, simultaneously with electro-precipitation of the biopolymers. For example, electrochemical deposition of various chitosan—multicomponent composites, e.g., carbon nanotubes, metal/metal oxide nanoparticles, enzymes, and polymers, have been reported for the purpose of biosensors. ${ }^{1,2,4-6,17}$

In this paper, we demonstrate that TOCN hydrogel can be electrochemically coated on the surface of any conductive material, even with complex $3 \mathrm{D}$ shapes. TOCN is a promising coating agent which enables not only forming its hydrogel but also single or multiple layered coatings consisting of micro or nanosized particles. Beyond biomedical applications, our recent discovery can be extended to fabrication of functional nanostructured materials for electrochemical energy devices. Such coating technique brings up new eco-friendly aqueousbased electrode synthesis methods for energy storage applications. Electrochemically precipitated TOCN-graphite composites were tested as negative electrodes in lithium ion batteries. TOCN contributes not only to the precipitation of graphite on the copper current collector but also acts as a binder of the graphite composites. Also, the electro-precipitated TOCN hydrogel itself can be applied in the field of hydrogen production as a protective layer against undesirable side reactions. The hydrogel deposited on the surface of a catalyst plays the role of a membrane, allowing HER to proceed, while preventing the simultaneously occurring oxygen reduction side reaction (ORR). To the best of our knowledge, this is the first time that electro-precipitation of TOCN hydrogel and its composite are introduced.

\section{EXPERIMENTAL SECTION}

Materials. Never dried cellulose pulp was supplied by Domsjö Fabriker AB (Domsjö, Örnsköldsvik, Sweden). TEMPO radical (2,2,6,6-tetramethylpiperidine-1-oxyl, free radical, 98\%) and lithium metal foil were purchased from Alfa Aesar. Triton X-100 (4-(1,1,3,3tetramethylbutyl)phenyl-polyethylene glycol) and sodium bromide $(\mathrm{NaBr})$ were purchased from Sigma-Aldrich, and sodium hypochlorite $(\mathrm{NaClO})$, sodium perchlorate $\left(\mathrm{NaClO}_{4}\right)$, perchloric acid $\left(\mathrm{HClO}_{4}\right)$, and polyethylenimine (PEI, M.W. 1800, branched) were from VWR international. Universal indicator solution $\mathrm{pH} 3-10$ was purchased from Fluka. Timrex SLP 30 AH-354 graphite and Super-P conductive carbon were kindly provided by Timcal Graphite \& Carbon. Carboncoated lithium iron phosphate $\left(\mathrm{LiFePO}_{4}, \mathrm{LFP}\right)$ of the type Life Power $\mathrm{P}_{2}$ (Phostech Lithium) was prepared. Also, $20 \mu \mathrm{m}$ thick copper foils (Advent research materials), 1 wt \% PTFE coated Toray carbon paper 060 (Toray), platinum $(\mathrm{Pt})$ plate, and copper and brass wires were used to demonstrate the feasibility of TOCN hydrogel coating techniques.

Preparation of TOCN. Oxidation of cellulose pulp was carried out in the same way as in a previous study, ${ }^{18}$ following the earlier reported protocols by Saito et al. ${ }^{19}$ First, $40 \mathrm{~g}$ (dry content) of never-dried pulp were washed with a $\mathrm{pH} 2 \mathrm{HCl}$ solution and suspended in deionized water, followed by mixing with TEMPO $(4 \mathrm{mmol})$ and $\mathrm{NaBr}(40$ $\mathrm{mmol}$ ). $\mathrm{NaClO}$ was slowly added to the suspension to achieve the desired amount of sodium carboxylated functional groups on the cellulose surface. The $\mathrm{pH}$ of the suspension was controlled and maintained at $\mathrm{pH} 10$ during the reaction using a $0.5 \mathrm{M}$ sodium hydroxide solution. The charge density of the TEMPO-oxidized cellulose pulp was determined to be $650 \mu \mathrm{mol} \mathrm{g}^{-1}$ (carboxylate content per unit mass of fiber) by conductometric measurements. ${ }^{20} 1$ wt $\%$ TEMPO-mediated oxidized cellulose pulp suspended in water was disintegrated by a microfluidizer (M-110EH, Micro fluidics Corp, United States), passed through two large chambers (400 and $200 \mu \mathrm{m}$ connected in series) at 925 bar for 3 times, and passed 3 times through the small chambers $(200$ and $100 \mu \mathrm{m})$, consecutively. The prepared 1 wt $\%$ TOCN gel with $650 \mu \mathrm{mol} \mathrm{g}^{-1}$ was diluted to $0.1 \mathrm{wt} \%$ aqueous suspension using an Ultra Turrax D125 Basic disperser at $10000 \mathrm{rpm}$ for $5 \mathrm{~min}$. The resulting dispersion is denoted as TOCN sol. Then $1 \mathrm{wt}$ $\%$ TOCN gel with higher charge density $\left(1550 \mu \mathrm{mol} \mathrm{g}^{-1}\right)$ was prepared in the same way as above and used for fabrication of lithium ion battery electrode.

Electro-precipitation of TOCN Based Hydrogel. Electroprecipitation of TOCN hydrogel was conducted using TOCN sol with different conductive materials as substrates (copper, brass wires, and carbon paper) and $0.1 \mathrm{wt} \%$ of TOCN aqueous suspension as the coating medium. The substrates were immersed in the coating medium and connected as anodes, and a Pt cage in the same container was used as counter electrode. Then an anodic current was applied for a certain time using a PAR273 potentiostat-galvanostat.

In this study a suitable current density range was found to be 1-10 $\mathrm{mA} / \mathrm{cm}^{2}$ to obtain a stable gel on the anode. A too high current density resulted in vigorous oxygen gas formation and the formed bubbles ripped off the gel and made coating impossible. On the other hand, a too low current density did not create the necessary $\mathrm{pH}$ gradient at the anode in a reasonable time. Details on current density and deposition time for the different experiments are given in the Results and Discussion.

In order to observe the $\mathrm{pH}$ profile of TOCN sol during electroprecipitation, TOCN sol mixed with $\mathrm{pH}$ indicator solution was prepared. Carbon paper $\left(1 \mathrm{~cm}^{2}\right)$, copper foil $\left(1 \mathrm{~cm}^{2}\right)$, and a platinum plate $\left(0.75 \mathrm{~cm}^{2}\right)$ were used as working electrodes with platinum as 
counter electrode. Electro-precipitation was performed by applying 1 $\mathrm{mA} / \mathrm{cm}^{2}$ of current density for $15 \mathrm{~s}$.

The growth of lateral thickness of hydrogels was measured varying the deposition time and the current densities, using a copper foil as the working electrode, whereas a platinum plate was used as the counter electrode ( $5 \mathrm{~mm}$ distance). The lateral thickness in the photographs of the gels was analyzed by the ImageJ software v1.51j8. ${ }^{21}$ Electropreiciptation of hydrogels on the substrates with complex 3D shapes was demonstrated using a copper wire and a brass wire. The hydrogels were freeze-dried to form aerogels thereof, and further characterized by infrared and energy-dispersive X-ray spectroscopy (EDX).

To show $\mathrm{pH}$ induced sol-gel transition of TOCN triggered by addition of acid directly to the sol, $50 \mu \mathrm{L}$ of $0.5 \mathrm{M} \mathrm{H}_{2} \mathrm{SO}_{4}$ was added to 1 $\mathrm{mL}$ of TOCN-sol.

Electro-precipitation of TOCN Hydrogel-Based Composites. To show the possibility of coating composite materials in one single synthesis step, an aqueous suspension containing $0.1 \mathrm{wt} \%$ of TOCN and $2 \mathrm{wt} \%$ of graphite was prepared as coating medium, followed by 20 min of ultrasonic treatment (Vibra-cell, Sonics). A copper wire, as working electrode, was immersed in the graphite-TOCN mixed dispersion and $9 \mathrm{~mA} / \mathrm{cm}^{2}$ of current density was applied for $30 \mathrm{~s}$. The hydrogel was frozen at $-196{ }^{\circ} \mathrm{C}$ using liquid nitrogen and freezedried for $24 \mathrm{~h}$ (Coolsafe 110-4, ScanVac) to a graphite-TOCN aerogel.

The growth of hydrogel composites was evaluated varying the deposition time and the current density, and the composition of the coating medium ( $0.1 \%$ TOCN with $2,0.95,0.5 \%$ of graphite). A copper foil $\left(1 \mathrm{~cm}^{2}\right)$ was used as the working electrode and the distance from a platinum counter electrode was $5 \mathrm{~mm}$.

Multilayered hydrogels were prepared by immersing bare and hydrogel coated substrates in different solutions and coating the medium electrochemically, similarly as above, on the top of the previous layer(s). A two-layered hydrogel, composed of a graphite containing hydrogel covered by a subsequently coated pure TOCN hydrogel, was fabricated on a copper plate $\left(j=2.5 \mathrm{~mA} / \mathrm{cm}^{2}\right.$ was applied for $30 \mathrm{~s}$ for each layer). Similarly, a three-layer composite was achieved by switching the coating medium for each layer. Lithium iron phosphate (LFP) was selected as a component due to its distinctive size distribution and elemental composition compared to that of graphite, so these can be easily distinguished by microscopic analysis and X-ray spectroscopy. The three layers consisted of a graphite/TOCN first layer, a LFP/TOCN second layer, and a graphite/TOCN third layer. The TOCN-LFP mixed suspension was prepared similarly as the graphite-TOCN suspension ( $2 \mathrm{wt} \%$ LFP and $0.1 \mathrm{wt} \%$ TOCN). A copper foil was used as the substrate and $10 \mathrm{~mA} / \mathrm{cm}^{2}$ current density was applied for $3 \mathrm{~s}$ to deposit each layer, on top of the previously coated layer, alternating the coating media. The electrode was dried in an oven at $80{ }^{\circ} \mathrm{C}$ for $30 \mathrm{~min}$ after each coated layer.

Preparation of Lithium Ion Battery Electrode. An aqueous suspension containing $2.35 \mathrm{wt} \%$ of graphite, $0.05 \mathrm{wt} \%$ of Super-P, and 0.1 wt $\%$ of TOCN with a charge density $1550 \mu \mathrm{mol} \mathrm{g}^{-1}$ was ultrasonicated for $20 \mathrm{~min}$ and used as the coating medium to fabricate graphite electrodes. The ratio of graphite/Super-P/TOCN was 94:2:4, as in our previous study, using the same materials. ${ }^{18}$ A $15 \mathrm{~mm}$ diameter of circular copper foil ( $20 \mu \mathrm{m}$ thickness) was employed as the working electrode, later used as the current collector in the lithium ion battery cell. Prior to electrochemical coating, 0.25 wt \% of PEI dissolved in methanol was spray coated to increase the adhesion between the graphite-TOCN composite and the copper foil. The PEI coated working electrode was immersed in the coating medium, and a Pt cage worked as counter electrode. The coating process was carried out by applying $11.3 \mathrm{~mA} / \mathrm{cm}^{2}$ current density for $10 \mathrm{~s}$, and subsequently the electrode was dried at $80{ }^{\circ} \mathrm{C}$ for $20 \mathrm{~min}$. Before the electrode was assembled to the battery cell, it was dried at $110^{\circ} \mathrm{C}$ in a vacuum dryer overnight. Selectilyte LP 40 from BASF, composed of ethylene carbonate (EC)/diethyl carbonate (DEC) 1:1 and $1 \mathrm{M} \mathrm{LiPF}_{6}$, was used as a liquid electrolyte with 2 wt \% of vinylene carbonate. The graphite electrode acted as working electrode and lithium foil was the counter electrode. They were assembled with a Celgard 2325 separator to a battery cell in an argon filled glovebox. The capacity of the aqueous processed graphite electrode was examined using a Solartron 1286
Electrochemical Interface potentiostat, controlled with the CorrWare software, at $0.048 \mathrm{C}$ of charging rate at the voltage range from 0.002 to $1.5 \mathrm{~V}$. The cycling performance of the graphite electrode was evaluated at constant current corresponding to $0.1 \mathrm{C}$ of charging rate at the initial charging.

Electro-precipitated TOCN Hydrogel on the Surface of the Pt Electrode As an ORR Protective Layer during HER. As a proof-ofconcept, a HER model study using a cocatalyst material widely used in photocatalysis was conducted. TOCN hydrogel was electrochemically precipitated on the surface of a $5 \mathrm{~mm}$ diameter Pt rotating disk electrode (RDE) in a 2-electrode setup using a Princeton Applied Research PAR273A potentiostat/galvanostat instrument. Before gel deposition, the Pt electrode was polished using $1 \mu \mathrm{m}$ sized alumina powder and a rotating polishing machine. Alumina particle residues were removed from the surface by ultrasonic treatment for $1 \mathrm{~min}$ in deionized water. A Pt cage was used as the counter electrode.

A mixture of 0.01 wt $\%$ of Triton X-100 and 0.1 wt $\%$ of TOCN was prepared in the same manner as the TOCN sol in the Preparation of TOCN section, denoted as TOCN-Triton X sol. The electroprecipitation of hydrogel was carried out by immersing a Pt RDE in the TOCN sol or TOCN-Triton $\mathrm{X}$ sol and applying $1 \mathrm{~mA} / \mathrm{cm}^{2}$ of anodic current for $1 \mathrm{~s}$. Then, the hydrogel coated bare Pt-RDE was transferred to a 3-electrode electrochemical cell where it was used as the working electrode, a Pt cage as counter electrode, and a silver/silver chloride $(\mathrm{Ag} / \mathrm{AgCl} /$ saturated $\mathrm{KCl})$ as the reference electrode. The electrochemical performance of the gel coated Pt electrodes as selective membranes during HER was further investigated by cyclic voltammetry (CV) and galvanostatic measurements, and compared with that of a bare platinum RDE. The $\mathrm{CV}$ and galvanostatic HER measurements were conducted using $\mathrm{pH}$ adjusted $1 \mathrm{M} \mathrm{NaClO}_{4}$ aqueous electrolytes saturated with $\mathrm{O}_{2}$, accomplished by bubbling $\mathrm{O}_{2}$ gas for 20 min prior to as well as during the $\mathrm{CV}$ and HER measurements. For the galvanostatic HER measurements, $j=-5$ or $-10 \mathrm{~mA} / \mathrm{cm}^{2}$ was applied for $10 \mathrm{~min}$ and the rotation rate of the Pt-RDE varied between 1000 and $2000 \mathrm{rpm}$. The current densities in industrial electrolysis are too high for a TOCN gel to be stable. If such high current densities are applied, vigorously evolving hydrogen gas will break the gel.

In order to compare the electrochemically active surface area (ECSA) of platinum, with and without surface coating, a CV study was carried out. ${ }^{22,23}$ A $0.1 \mathrm{M} \mathrm{HClO}_{4}$ aqueous solution was purged with nitrogen gas and the potential swept in the range between -0.25 and $+0.8 \mathrm{~V}$ vs $\mathrm{Ag} / \mathrm{AgCl}$, without rotating the $5 \mathrm{~mm}$ circular Pt electrode (scan rate $50 \mathrm{mV} / \mathrm{s}$ ). To evaluate the $\mathrm{H}_{2}$ oxidation on the Pt $\mathrm{RDE}$, in the presence/absence of the TOCN protective layer, linear sweep voltammetry (LSV) was performed in the range of -0.4 to $+0.6 \mathrm{~V}$ vs $\mathrm{Ag} / \mathrm{AgCl}$ reference electrode, bubbling hydrogen gas at $10 \mathrm{~mL} / \mathrm{min}$ flow rate (scan rate $10 \mathrm{mV} / \mathrm{s}$ ) in $1 \mathrm{M} \mathrm{NaClO}_{4}$ electrolyte. To examine the permeability of ferricyanide, a $\mathrm{CV}$ study was conducted in the potential range between -1.0 and $+0.5 \mathrm{~V}$ vs $\mathrm{Ag} / \mathrm{AgCl}$, using $1 \mathrm{M}$ $\mathrm{NaClO}_{4}$ solution containing $10 \mathrm{mM}$ of $\mathrm{K}_{3} \mathrm{Fe}(\mathrm{CN})_{6}$ (scan rate $10 \mathrm{mV}$ / s).

Physical Characterization of the Samples. A PerkinElmer Spectrum 100 Fourier-transform infrared spectrometer was used to investigate the mechanism of electro-precipitation of TOCN. Hydrogels on different types of conductive substrates were freeze-dried and then examined using attenuated total reflection (ATR) mode in the range of $600-4000 \mathrm{~cm}^{-1}$ with $4 \mathrm{~cm}^{-1}$ resolution. The composition of the freeze-dried graphite/TOCN aerogel was also analyzed using thermogravimetric measurement (TGA, TGA/DSC 1, Mettler Toledo). Microscopic images of the dried samples were obtained by field emission scanning electron microscopy (FE-SEM, S-4800, Hitachi) operated at $1 \mathrm{kV}$ accelerating voltage. To analyze the composition of the coatings, EDX (X-Max 80 SDD, Oxford Instruments) was used at $15 \mathrm{kV}$ accelerating voltage.

\section{RESULTS AND DISCUSSION}

Electrochemical Precipitation of TOCN Hydrogel on Conductive Substrates. Previous studies of $\mathrm{pH}$ induced solgel transition process of TOCN were typically performed by 
adding an acid to a TOCN suspension in a certain container with a fixed shape. ${ }^{13,15}$ The same $\mathrm{pH}$ induced sol-gel transition of TOCN can be induced by the local $\mathrm{pH}$ decrease on an electrode surface during electrochemical reactions, e.g., water oxidation (Figure 2).
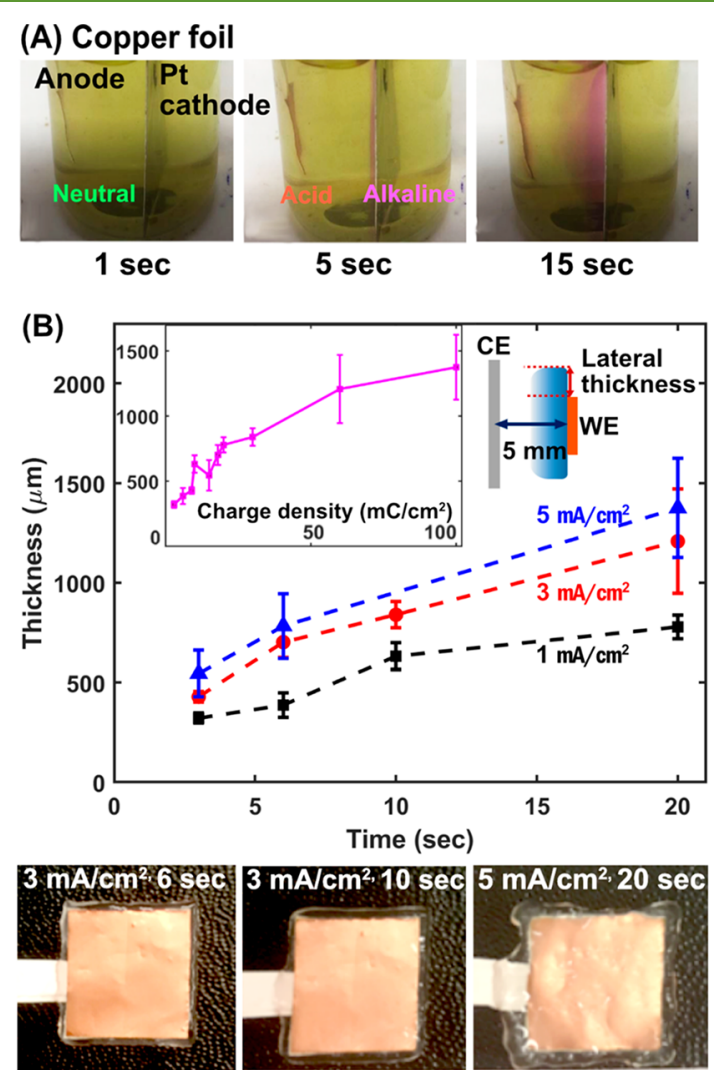

Figure 2. (A) Photographs of water splitting caused local $\mathrm{pH}$ change induced TOCN sol-gel transition with universal $\mathrm{pH}$ indicator (red when $\mathrm{pH}<3$, pink when $\mathrm{pH}>10)$, as a function of time $(1,5$, and $15 \mathrm{~s})$ on a copper foil anode. (B) Time dependent thickness growth as a function of current density $\left(1,3\right.$, and $\left.5 \mathrm{~mA} / \mathrm{cm}^{2}\right)$ and time (up to $20 \mathrm{~s}$ ) and photographs of the gels made at the given deposition conditions

Growth of the $\mathrm{pH}$ gradients at the anode using TOCN sol mixed with universal $\mathrm{pH}$ indicator (red at $\mathrm{pH}<3$, yellow at $\mathrm{pH}$ 4, light green at $\mathrm{pH} 5-7$, dark green at $\mathrm{pH} 8-9$, pink at $\mathrm{pH}>10$ ) was documented as photos (Figures $2 \mathrm{~A}$ and $\mathrm{S1A}, \mathrm{B}$ ) and Supporting Information (SI) videos. During water splitting and simultaneously occurring sol-gel transition of TOCN, color changes near the surfaces of the electrodes were observed: red at the anode and pink at the cathode, while bulk was neutral (Figures 2A and S1A,B). The SI videos also demonstrate how protons propagate away from the anodic surface, while hydroxide move from the cathode. It explicitly shows the localized $\mathrm{pH}$ changes and diffusion of proton and hydroxide ions generated from anode and cathode, respectively. Compared to carbon and platinum, the color change near the copper electrode propagated much less, which is due to two reactions occurring in parallel: oxygen evolution and copper dissolution.

The gelation of TOCN proceeds as the concentration of protons increase and cause the gelation of the TOCN in the vicinity of the anode. As shown in Figure 2B, the thickness of the gel increases with the time and applied current density. However, the growth rate of the hydrogel thickness decreases when the charge density is higher than $30 \mathrm{mC} / \mathrm{cm}^{2}$. In addition, the standard deviation of thickness increases due to flocculation of the hydrogel.

The advantage of this electrochemical precipitation is the freedom of the experimental setup, which is not limited to the shape of the container, rather directly immobilizing hydrogel on the surface of conductive substrates with complex-shapes. ${ }^{1,4,5,11}$ In addition, the growth of the hydrogels can be controlled by adjusting the operating variables, e.g., current, voltage, or time. The electrochemically coated hydrogels maintain good affinity with the coated substrate surface in the wet state.

Sol-gel transition of TOCN can be induced by acid addition directly to the sol. Figure S2B,C shows aggregation of TOCN to hydrogel. However, a firm hydrogel with cylindrical shape was not formed but rather flocculated. To make a firm hydrogel, it requires the addition of additional salts or cross-linkers or an increase in the concentration of TOCN in the aqueous dispersion, as suggested in refs 9, 13, and 24.

As shown in Figure 3A-D, TOCN hydrogels can be coated on different conductive materials. Geng et al. ${ }^{1,11}$ reported similar electrodeposition of chitosan and carboxymethyl cellulose (CMC) hydrogels by metal coordinated gelation. These could be deposited on copper and silver but not on graphite or platinum. In that case the dissolved metal ions induced the gelation of chitosan not the local $\mathrm{pH}$ change. On the contrary, electro-precipitation of TOCN does not require additional cross-link forces from metal ions and is possible on a wide range of substrates with electrical conductivity. This was proven by performing the electro-precipitation studies on substrates where no, or only minor, metal dissolution occurs (e.g., carbon paper and platinum).

To confirm the $\mathrm{pH}$ induced gelation, functional groups in the surface of $\mathrm{TOCN}-\mathrm{COO}^{-} \mathrm{Na}^{+}$and $\mathrm{TOCN}-\mathrm{COOH}$ were analyzed by FT-IR spectroscopy, as shown in Figure 3E. The FT-IR spectra show the level of protonation of freeze-dried electro-precipitated TOCN hydrogels (aerogels), formed on different substrates. As a reference, the aerogel made of pristine TOCN was also studied (denoted as "No template" in the legend). In this case, it showed a peak at $1607 \mathrm{~cm}^{-1}$, confirming the presence of deprotonated carboxyl groups in the structure (carbonyl absorption in the $\mathrm{COO}^{-} \mathrm{Na}^{+}$). This peak can be observed for the aerogels electro-precipitated on different substrates as well. However, for these samples, another peak at $1724 \mathrm{~cm}^{-1}$ can be seen, which corresponds to the stretching of carbonyl groups of the protonated carboxylate groups $(\mathrm{COOH})$.

Looking at the intensity ratio of these peaks, which is related to the proportion of the protonated and deprotonated carboxyl groups, a significant difference can be observed between the deposits formed on a copper substrate and that formed on carbon paper, see Figure 3E. As for the latter, the peak at 1724 $\mathrm{cm}^{-1}$ is significantly more intense than that for copper, indicating that the gelation of TOCN was accomplished only by charge neutralization due to protonation, without any other components inducing inter- or intra fibril cross-linking of TOCN. Even though platinum can be dissolved slowly at oxidative potentials, ${ }^{25}$ the gel formed on this substrate shows a very similar level of protonation to that of the gel formed on carbon paper. This indicates that the gel formation is mostly driven by the $\mathrm{pH}$ gradient. For the gel on copper, the peak at $1724 \mathrm{~cm}^{-1}$ is much less intense than that at $1607 \mathrm{~cm}^{-1}$, implying that a smaller portion of the carboxyl groups are protonated in this case. It is therefore assumed that the gel on copper was formed by two processes occurring in parallel: (1) $\mathrm{pH}$ induced gelation and (2) copper ion mediated cross-linking. Nonethe- 

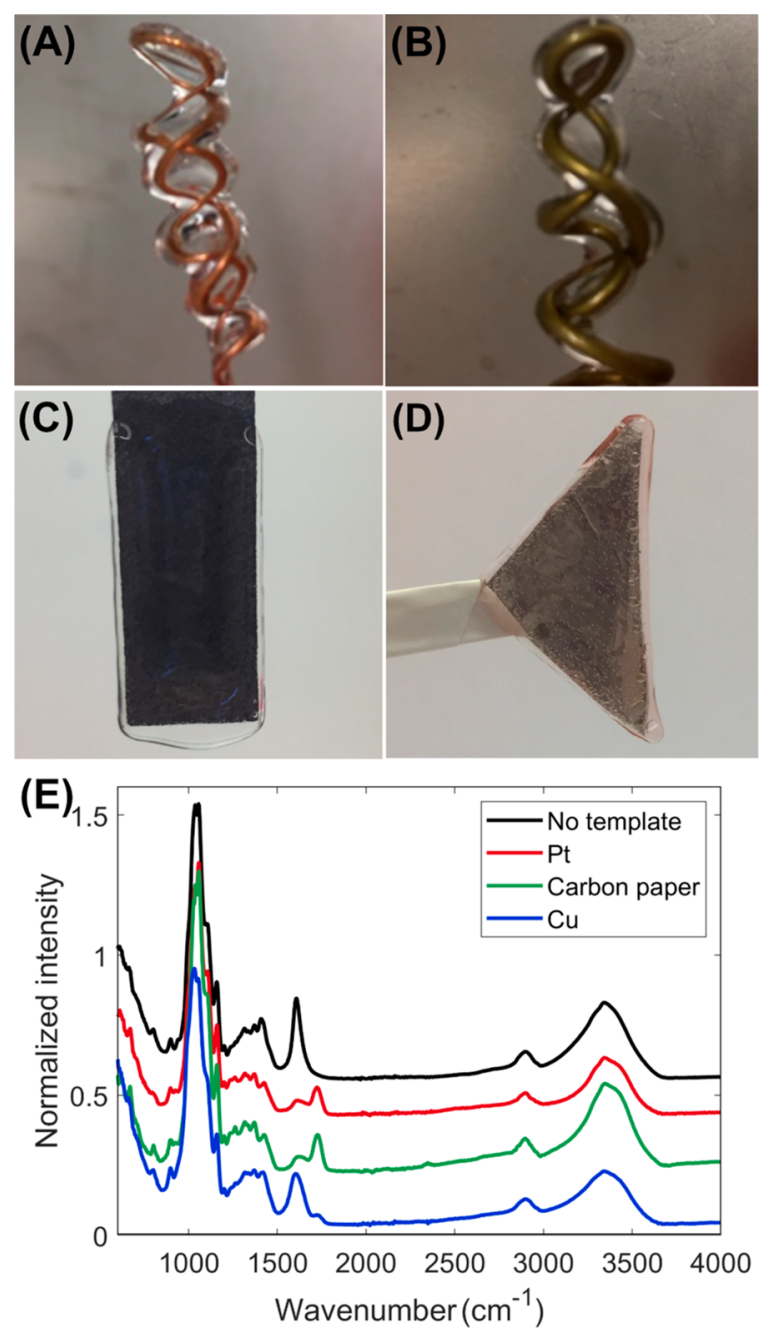

Figure 3. (A-D) Anodically electro-precipitated TOCN hydrogels on conductive substrates, (A) copper wire $(I=10 \mathrm{~mA}, t=60 \mathrm{~s})$, (B) brass wire $(I=5 \mathrm{~mA}, t=60 \mathrm{~s}),(\mathrm{C})$ carbon paper $(I=10 \mathrm{~mA}, t=20 \mathrm{~s})$, (D) platinum plate $(I=2 \mathrm{~mA}, t=15 \mathrm{~s})$, and (E) FT-IR spectra of freezedried hydrogels of pristine TOCN (no template) as well as electrochemically precipitated on different conductive substrates (platinum plate, carbon paper and copper foil). In panel E, the spectra were shifted along the $y$-axis for better visibility.

less, an EDX study of a "Cu" aerogel showed only 0.7 atomic wt $\%$ of copper content (the rest of elements were $57 \%$ of $\mathrm{C}$ and $42 \%$ of $\mathrm{O}$ ). Compared to the ion coordinated gelation of chitosan, containing $7 \%$ of $\mathrm{Cu}^{1}$, or $\mathrm{CMC}$ with $13.5 \%,{ }^{11}$ it is significantly lower in our case.

Electro-precipitation of TOCN Gels and Nanoparticles. Electrochemical deposition of biopolymer-based composite coatings has been studied using a variety of materials including carbon nanotubes, metal/metal oxide nanoparticles, and enzymes. ${ }^{1,2,4-6,17}$ Such polymers work as binders, adhering nanomaterials dispersed in the coating medium and allowing their fixation on the target substrates. In this respect, TOCN can also be regarded as a promising coating agent to fabricate hydrogel/aerogel composites, including functional materials, by a one-pot electro-precipitation technique.

An aqueous solution containing $2 \%$ of graphite particles and $0.1 \%$ of TOCN was prepared and electrochemical coating of the hydrogel thereof was conducted using a copper wire electrode $(\mathrm{j}$ $\left.\approx 9 \mathrm{~mA} / \mathrm{cm}^{2}, t=30 \mathrm{~s}\right)$. The obtained coating was freeze-dried to maintain its dimensional integrity. Graphite with $10-20 \mu \mathrm{m}$ particle size could be coated together with TOCN hydrogel on the copper coil, as shown in Figure 4 A. It also shows that TOCN
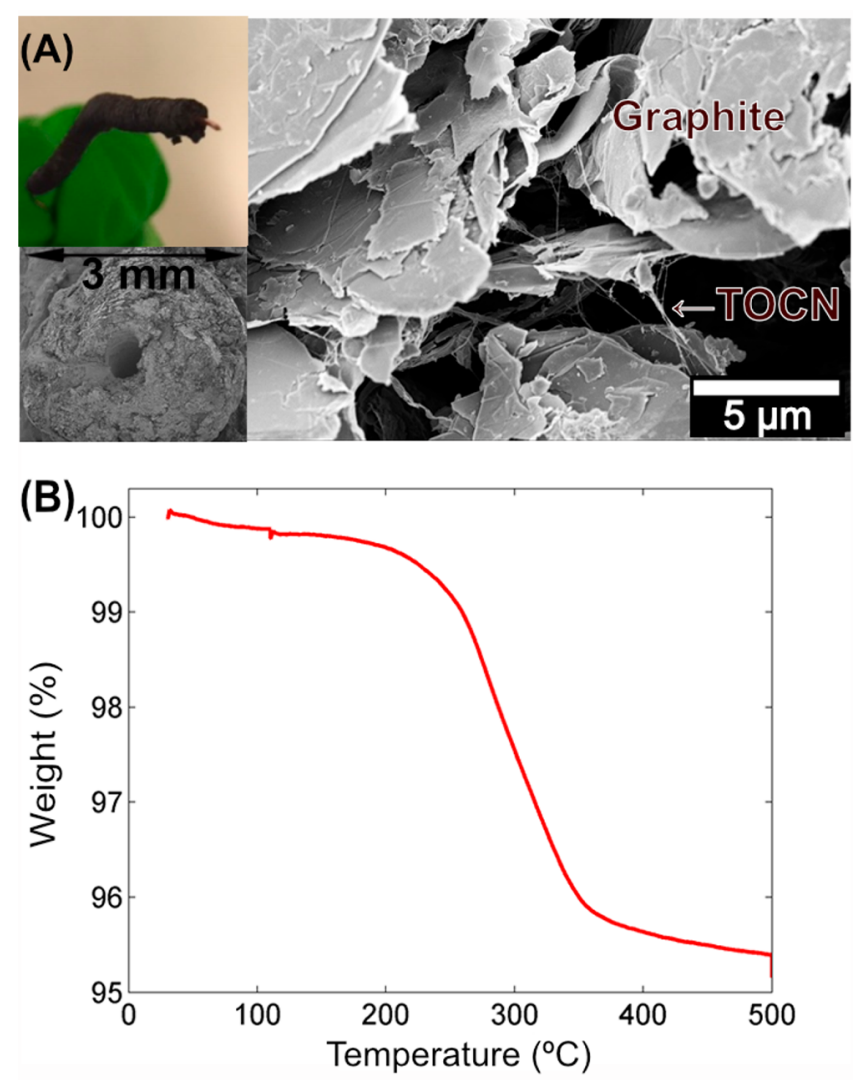

Figure 4. (A) Images of TOCN-graphite aerogel composite prepared by electro-precipitation of $2 \mathrm{wt} \%$ graphite and $0.1 \mathrm{wt} \%$ TOCN mixed dispersion on a copper wire, followed by freeze-drying and its microscopic image, the scale bar $5 \mu \mathrm{m}$ (B) composition analysis of the graphite-TOCN aerogel by TGA.

works as a binder, gluing not only TOCN hydrogel on the surface of the template, but also the carbon particles. Such beneficial properties of TOCN were observed in our previous studies showing its feasibility as a binder to fabricate composites by vacuum-filtration with high dimensional stability for further applications. $^{18,26}$

The composition of the composite was investigated by thermogravimetry (Figure 4B). A $4.85 \%$ of weight loss up to 500 ${ }^{\circ} \mathrm{C}$ corresponds to thermal degradation of TOCN, ${ }^{18}$ meaning that the composite consists of 5\% of TOCN and $95 \%$ of graphite (ratio 1:19), a ratio similar to that in the aqueous dispersion. This result indicates that the composition of an electroprecipitated TOCN composite can be simply tuned by the composition of the coating medium. While the $\mathrm{pH}$ responsive TOCN is aggregated, it can embrace the particles adjacent to the fibrils and trap them in the gel matrix. It is interesting that nanosized cellulose fibrils form a firm gel holding microsized particles, dimensionally more than 100 times larger and quantitatively at 20 times higher concentration, than that of TOCN. Compared to the studies of electrodeposition of biopolymer-nanoparticle composites, e.g., polymers, carbon nanotubes, graphene sheets, and metal/metal oxide nanoparticles, ${ }^{1,2,4-6,17}$ our present study demonstrates the possibility 
$\mathbf{1}^{\text {st }}$ layer coating
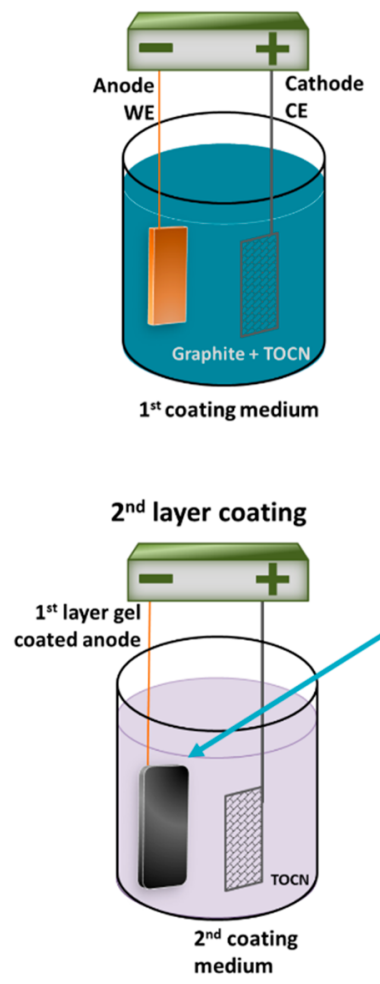

Aggregation of TOCN including adjacent particles at the anode surface

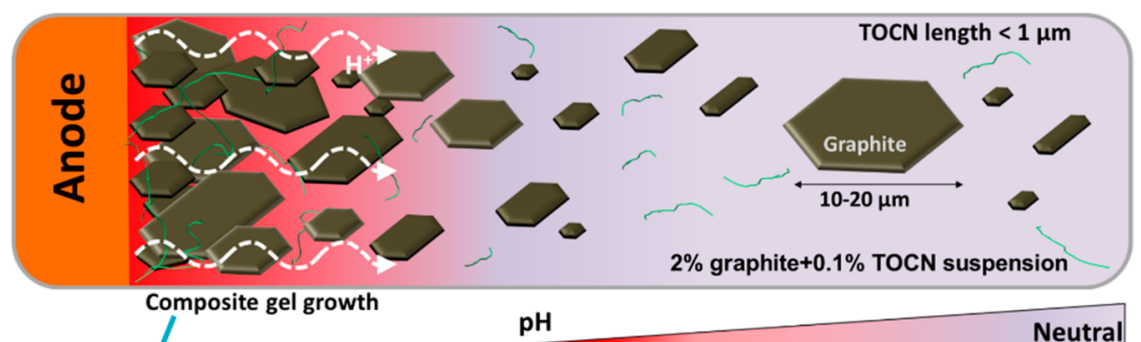

Neutral

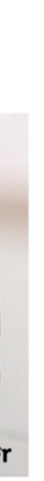

$1^{\text {st }}$ layer
Graphite+TOCN $2_{\text {TOCN }}^{\text {nd }}$ layer

$0.1 \%$ TOCN suspension

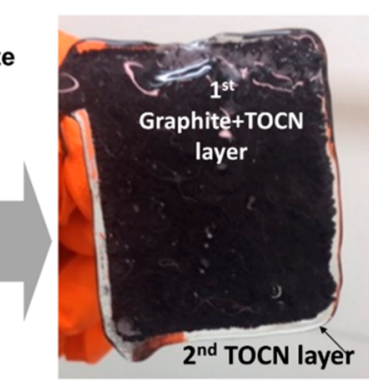

Figure 5. Schematic description of fabrication of multilayer hydrogel composite materials on conductive substrates by electrochemical precipitation.

of coating distinctively larger, micrometer-sized, particles on desired substrates.

The thickness and the mass loading of the composites increase as a function of time and current applied, in the same manner with TOCN based hydrogel, as shown in Figure S3A,B. Figure $\mathrm{S} 3 \mathrm{C}$ demonstrates a poor coverage of graphite particles on the substrate surface when a current density that is too low $(<3 \mathrm{~mA} /$ $\mathrm{cm}^{2}$ ) was applied for very short time, compared to that of higher values $\left(>5 \mathrm{~mA} / \mathrm{cm}^{2}\right)$ for longer deposition time, as shown in Figure S3D. Figure S3E showed the compositions of the composites can be controlled depending on the concentration of the coating medium. The concentration of the incorporated microparticles in the deposited composite gel reflected the composition of the sol used for the electrodeposition within $\sim 2 \%$.

To visualize the possibility to deposit multiple layers on top of each other, two sequential coatings with different coating media were carried out (Figure 5); one with $0.1 \mathrm{wt} \%$ TOCN with $2 \mathrm{wt}$ $\%$ graphite, the other with $0.1 \mathrm{wt} \%$ TOCN. To construct the first layer, the copper plate was immersed in the first coating medium (TOCN/graphite mixture) and $j=2.5 \mathrm{~mA} / \mathrm{cm}^{2}$ of current was applied for $30 \mathrm{~s}$. Afterward, the TOCN/graphite hydrogel coated copper plate was dipped in the second coating medium and the TOCN gel was coated on top.

A trilayered composite was also constructed by the same technique as above. Two different coating media were prepared to visualize the separated coating layers; one was a graphite containing TOCN suspension, while the other was an LFP containing TOCN suspension. Sequential layers were deposited in the order of graphite-LFP-graphite containing hydrogels. LFP was employed because of its distinctively different size distribution (particle size ranged 200-500 nm Figure S4) and elemental composition ( $\mathrm{Li}, \mathrm{Fe}, \mathrm{P}, \mathrm{O})$, compared to that of graphite (particle size $10-20 \mu \mathrm{m}$, as shown in Figure 6A). Figure S4, the magnified microscopic image of the second layer of trilayered composite demonstrates TOCN binding LFP particles. The microscopic image in Figure 6A shows wellordered multiple layers. The oven-dried trilayer composite showed three separated layers, thus the hydrogel layers did not mix or collapse during drying. The dimensional integrity of each layer and of the final product composite were well maintained even after drying. The structure was very stable not only in the wet hydrogel but also in the dried form with $\sim 10 \mu \mathrm{m}$ thickness of the LFP containing layer and $30 \mu \mathrm{m}$ thickness of the two graphite containing layers. The thickness difference is attributed to the size difference of LFP and graphite particles.

The elemental mapping images in Figure 6B explicitly show that the coating layers were well separated. The elemental composition of the trilayered composite was $90 \%$ of C, $8.3 \%$ of $\mathrm{O}, 0.6 \%$ of $\mathrm{Fe}, 0.5 \%$ of $\mathrm{P}$, and $0.3 \%$ of $\mathrm{Cu}$. The line scan measurements of chemical elements were carried out at the position where the yellow line is drawn in Figure 6A. The intensities of each element are displayed as a function of distance between two points on the yellow line. The distance between the start point (set as 0 in Figure 6A and on the $x$-axis in Figure 6B) and the end point (denoted as 80 in Figure $6 \mathrm{~A}$ and the end point of $x$-axis in Figure $6 \mathrm{~B})$ is $80 \mu \mathrm{m}$. The first and third layers, composed of graphite- TOCN (19:1) layer, contain only a little amount of oxygen, iron and phosphorus but high amounts of carbon. Intensities close to zero, shown in the line scan EDS results of carbon, were because of the porous structure. The second layer with LFP-TOCN contains high amounts of oxygen, iron and phosphorus, and a lower amount of carbon. This qualitative study using EDS shows that this electro-precipitation of TOCN containing suspensions enables facile fabrication of 


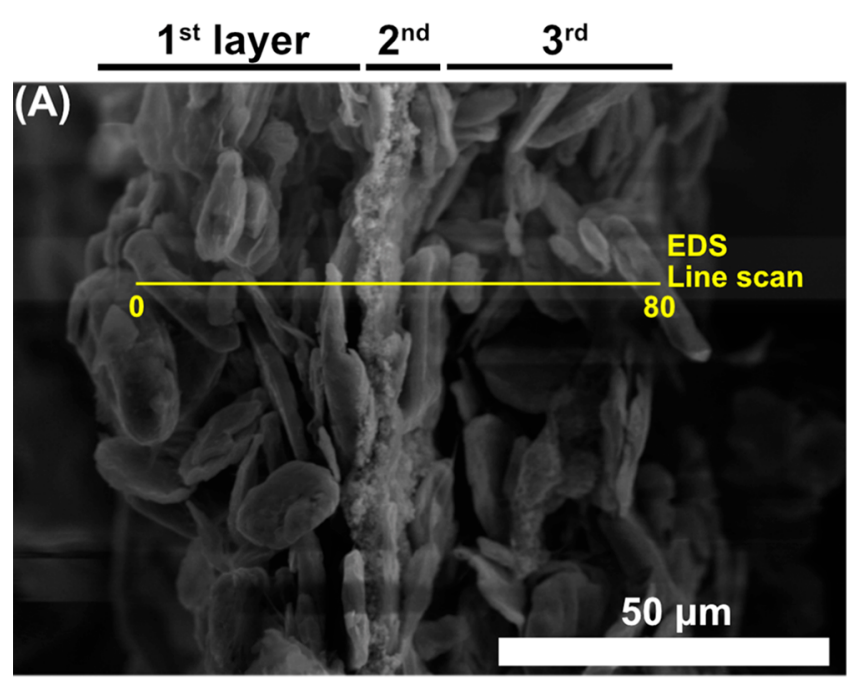

(B)
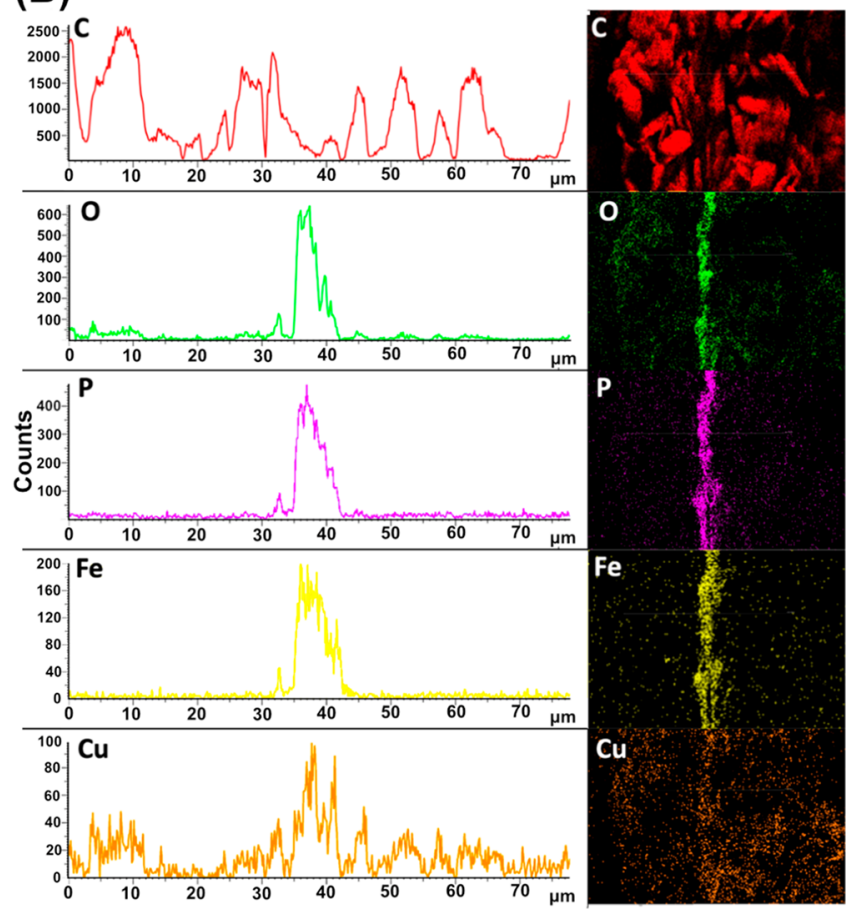

Figure 6. EDS spectrum by line scanning and quantification of element components, (A) microscopic images of electro-precipitated trilayered composite, the yellow line is the position where the line scan EDS measurements were carried out. (B) EDS line scan and mapping images of various element components in the composite.

multiple layered-hydrogels/composites of high dimensional stability.

We emphasize that not only graphite and LFP, but also other water dispersible particles of nano-or micrometer size, can be coated with $\mathrm{pH}$ responsive TOCN. The method is very practical. However, its limitations are mainly due to the colloidal stability of the TOCN. The concentration in water is limited to $0.2 \%$. Regarding the deposition of a composite, the components must be stable in the $\mathrm{pH}$ and potential ranges used during the experiments. Another limitation is the necessity of an electronically conducting substrate.

Furthermore, as the dispersion is prepared in water, it can be considered as an environmentally friendly process. It is expected that such a coating technique can widen the spectrum of applications of TOCN as coating agent. For example, the tightly bound but porous structure of the composite shown in Figures 5 and 6 is a desirable property for electrodes in electrochemical energy devices, e.g. lithium ion batteries. Thus, such applications were further investigated as detailed in the following sections.

Aqueous Based Electrochemical Deposition of Graphite for the Negative Electrode of Lithium Ion Batteries. State-of-the-art electrode fabrication processes for lithium ion batteries require the usage of environmentally harmful and toxic solvents, e.g. 1-methyl-2-pyrrolidinone (NMP). Aqueous processes carried out by filtration of aqueous suspension containing active materials and binders have been suggested, ${ }^{18,26,27}$ but these require an extra vacuum filtration setup and water drainage, and takes tenths of minutes. Electrophoretic deposition relies on the electrophoretic mobility of materials and requires the application of high voltage. ${ }^{28-30}$ For example, Lalau et al. $^{28}$ reported that electrophoretic deposition of $\mathrm{LiN}_{1 / 3} \mathrm{Mn}_{1 / 3} \mathrm{Co}_{1 / 3} \mathrm{O}_{2}$ was carried out at $80 \mathrm{~V}$ constant voltage applied for $5 \mathrm{~min}$ using an NMP-based colloidal coating medium. Seo et al. ${ }^{31}$ produced carbon nanotube/ graphene nanosheet composites by electrophoretic deposition, applying $300 \mathrm{~mA}$ of a constant DC current for $1 \mathrm{~h}$, and applied it as a negative electrode in a lithium ion battery. In this respect, by using TOCN as binder, the currently studied electrocoating technique is beneficial to coat the carbon active material in a short period of time at significantly milder conditions.

The images of graphite electrodes made by electroprecipitation of a graphite containing TOCN hydrogel composite and its microscopic image are displayed in Figures $7 \mathrm{~A}$ and $\mathrm{B}$, respectively). By applying $11 \mathrm{~mA} / \mathrm{cm}^{2}$ current density for $10 \mathrm{~s}, 11 \mathrm{mg}$ of composite was obtained on the surface of a circular copper template, which was further used as current collector. We note that it was important to pretreat the copper electrode with a thin, spray-coated PEI layer, which was an excellent adhesive to increase the dimensional stability of the graphite-TOCN composite on copper during and after drying. As shown in Figure 7A, the graphite-TOCN composite was fixed on the copper foil without fractures, even when it was bent. The electrochemical polarization profiles of the graphite electrode at different charging rates are shown in Figure $7 \mathrm{C}$. The voltage profile of the graphite electrode was similar to that of typical graphite electrodes, showing the plateaus at the different intercalation stages below $0.2 \mathrm{~V}^{32,33}$ At $0.048 \mathrm{C}$ of charging rate $\left(0.085 \mathrm{~mA} / \mathrm{cm}^{2}\right), 320 \mathrm{mAh} / \mathrm{g}$ charge capacity was measured, which is only slightly lower than the theoretical capacity of graphite $(372 \mathrm{mAh} / \mathrm{g})$. The galvanostatic cycling performance of the graphite electrodes was examined over 20 cycles, applying $0.14 \mathrm{~mA} / \mathrm{cm}^{2}$ of a constant current which corresponds to $0.1 \mathrm{C}$ of charging (a full charging or discharging takes $10 \mathrm{~h}$ ) rate in the beginning of the test (Figure 7D). It showed $260 \mathrm{mAh} / \mathrm{g}$ of the initial specific capacity and $162 \mathrm{mAh} /$ $\mathrm{g}$ after 20 cycles. Compared to our previous study using the same materials with the same ratio of active and binder materials, ${ }^{27}$ the graphite electrode prepared by electro-precipitation showed somewhat lower capacity. Nevertheless, by optimizing the composition further (e.g., lowering the content of PEI and TOCN), we expect that the capacity can increase, and that this technique might be an appealing, environmentally friendly way of forming electrodes for different kind of batteries.

Electrochemically Coated Hydrogel As a Selective Membrane for HER. With increased demand for clean energy, hydrogen has been considered as a promising energy carrier for renewable energy technologies, as a possible replacement of 

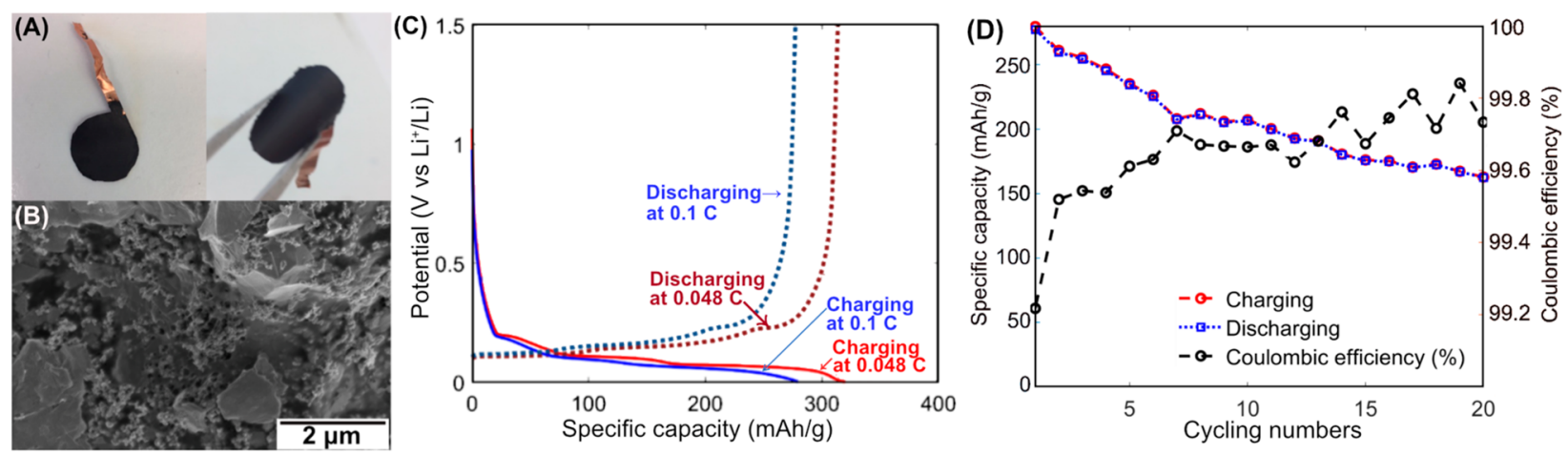

Figure 7. (A) Images of graphite electrode coated on the PEI-coated copper electrode, (B) microscopic image of the graphite, (C) capacity measurements of graphite electrode at 0.048 and $0.1 \mathrm{C}$ of charging rates $(2 \mathrm{mV}-1.5 \mathrm{~V}$ of potential window) and (D) galvanostatic charging and discharging cyclic stability test of the cell at $0.1 \mathrm{C}$ charge/discharge rate.

fossil fuels. ${ }^{34}$ Photocatalytic water splitting is one promising future hydrogen production technique. To improve its efficiency, the backward reaction of recombination of oxygen and hydrogen to water on the cocatalyst surface must be circumvented. $^{35}$ Here as a proof-of-concept study, we investigated the hydrogen evolution reaction (HER) selectivity in the absence/presence of oxygen on platinum (a typical cocatalyst in photocatalysis) electrode coated with selective membrane.

Figure 8A shows a schematic description of HER with and without a barrier layer against ORR, which is an unwanted side

\section{(A)}

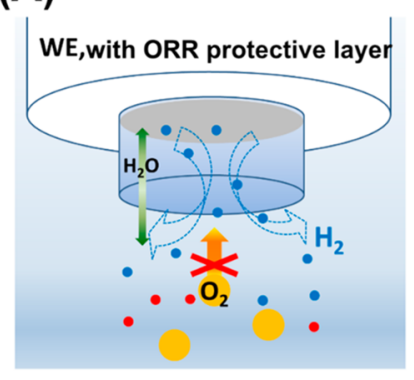

(B)

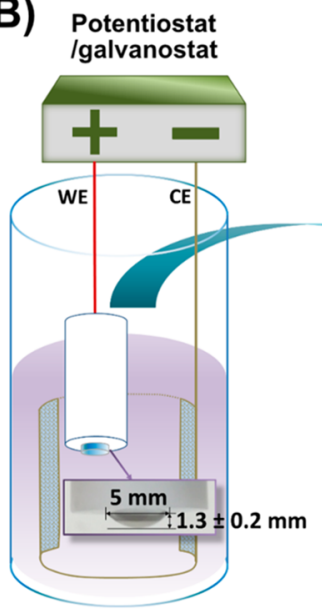

Coating
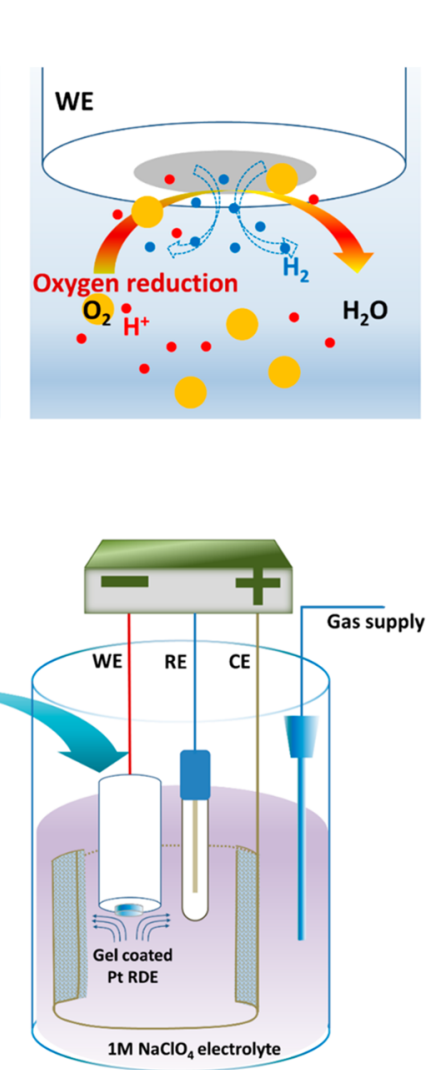

Electrochemical measuremenets

Figure 8. (A) Schematic illustrations of HER with/without ORR protective layer (B) the electrochemical coating and measurement setups.

reaction in this case. To prevent such loss, there have been extensive studies about protective coatings, mostly composed of amorphous oxide layer including chromium oxide, ${ }^{35-37}$ titanium dioxide, $^{38}$ nickel oxide, ${ }^{39,40}$ silicon dioxide, ${ }^{41-43}$ and vanadium oxide. ${ }^{44}$ Other than those, biopolymer-based hydrogels may be possible candidates as ORR protective layers, if permeable for water and smaller ions, but barriers for oxygen as shown in Figure 8A.

The electrochemical cell for the coating of the TOCN gel (two electrodes setup denoted as "Coating") and the further electrochemical measurements for the $\mathrm{CV}$ and the galvanostatic HER (three electrodes setup denoted as "Electrochemical measurements") are depicted in Figure $8 \mathrm{~B}$. The TOCN hydrogel was coated on a static Pt disk electrode. Applying $j=$ $1 \mathrm{~mA} / \mathrm{cm}^{2}$ for $t=1 \mathrm{~s}$ was found as the best condition to form a stable TOCN gel. The TOCN sol was freshly prepared, before the experiments, and stored at room temperature. Note that using the same dispersion for multiple electro-precipitation experiments did not lead to reproducible results.

To further increase the stability of the gel (e.g., the adhesion of the hydrogel on the Pt surface), Triton X-100 surfactant was added to the deposition solution. To avoid micelle formation, the concentration of Triton X-100 was kept below its critical micelle concentration $(0.22 \mathrm{mM})$, at 0.01 wt $\%$. The TOCNTriton X-100 sol was used as a coating medium, and the hydrogel was precipitated similarly as above.

The hydrogel coated $\mathrm{Pt}$ disk was transferred from the coating cell setup to the 3-electrode setup with an $\mathrm{O}_{2}$ saturated $1 \mathrm{M}$ $\mathrm{NaClO}_{4}$ electrolyte, as described in Figure $8 \mathrm{~B}$, for further electrochemical measurements. The gel showed good stability at slightly acidic and neutral conditions, whereas, at alkaline conditions, the gel was easily destroyed during HER and had no effect on ORR prevention.

In Figure 9A, on bare platinum, a current plateau can be observed between -0.2 and $-1 \mathrm{~V}$, related to the diffusion limited ORR at rotating rate of $1000 \mathrm{rpm}$. As for the hydrogel coated Pt electrodes, both the bare TOCN and the TOCNTriton X hydrogel coatings led to a significant decrease of the ORR current. It was more pronounced with the Triton X-100 containing hydrogel, which indicates that the surfactant contributes to better coating of the Pt electrode (and/or further blocking the $\mathrm{O}_{2}$ transport). Although preventing the ORR, HER could take place on the gel coated electrodes at potentials below $-0.8 \mathrm{~V}$. An oxidation peak appeared on the backward anodic scan at $-0.8 \mathrm{~V}$, which can be related to the back-oxidation of the formed and trapped $\mathrm{H}_{2}$. This indicates that HER takes place at 

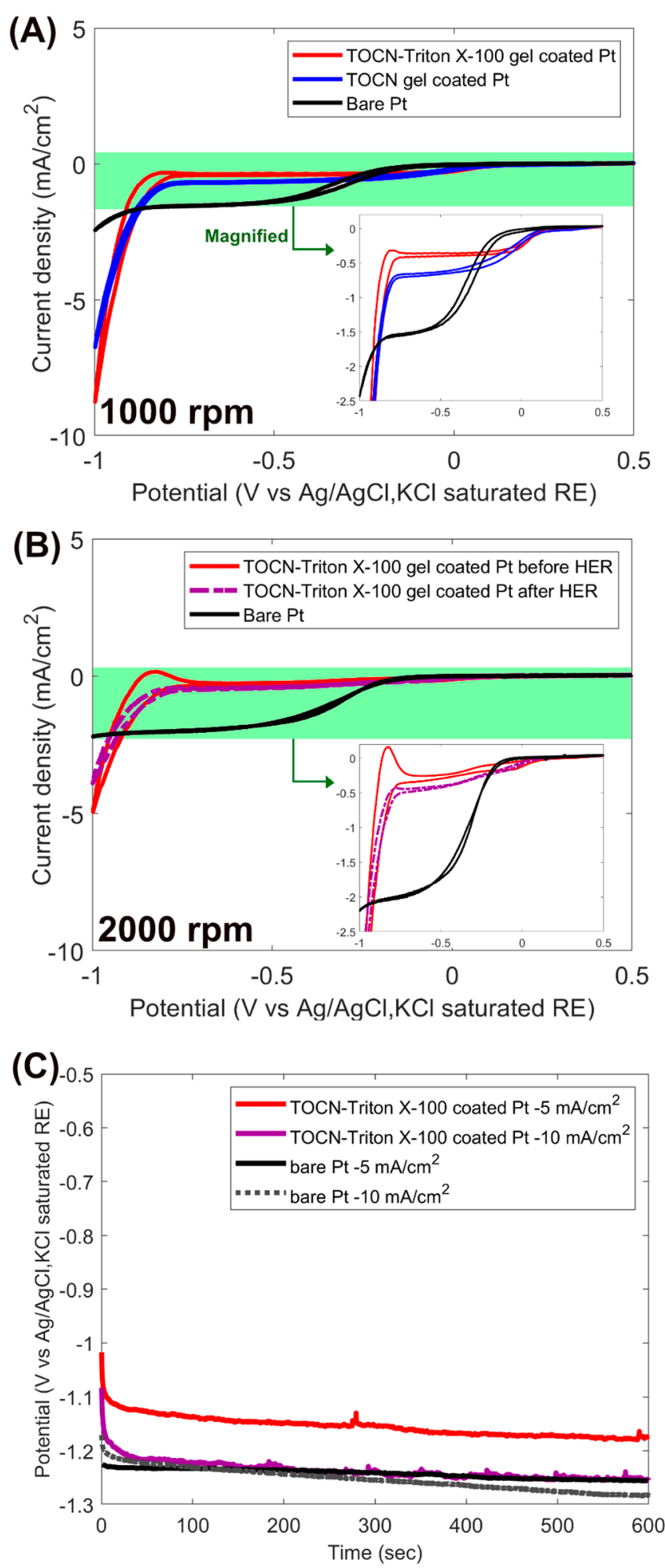

Figure 9. Electrochemical measurements of gel coated Pt electrodes in $\mathrm{O}_{2}$ saturated $\mathrm{pH} 4 \mathrm{NaClO}_{4}$ solution at rotating speed of (A) 1000 and (B) $2000 \mathrm{rpm}$, the magnified view of the green colored region $(j=$ $-2.5-0.5 \mathrm{~mA} / \mathrm{cm}^{2}$ ) as shown in the right side (scan rate $10 \mathrm{mV} / \mathrm{s}$ ). (C) galvanostatic HER measurement of TOCN-Triton X-100 gel coated Pt electrode at -5 and $-10 \mathrm{~mA} / \mathrm{cm}^{2}$ at rotating rate of $2000 \mathrm{rpm}$.

the interface between Pt-RDE and TOCN gel, hence water can penetrate the gel, whereas $\mathrm{O}_{2}$ cannot and some $\mathrm{H}_{2}$ is also being trapped. The stability of the layers was initially addressed by performing a constant current electrolysis at $j=-5 \mathrm{~mA} / \mathrm{cm}^{2}$ current density with the gel coated electrodes (Figure 9C) and recording a cyclic voltammogram on the used electrodes to compare this with the initial results (Figure S5).

Two possible reasons for the onset of ORR/HER at a more positive potential on a coated cathode than on bare Pt are (1) suppressed formation of oxide layer with the presence of TOCN hydrogel and (2) a lower $\mathrm{pH}$ in the hydrogel than in the bulk solution. It has been reported that coating a electrode suppressed the growth of platinum oxides and the onset potential of its oxygen reduction shifted to a more positive potential than that of the platinum without the surface coating. ${ }^{45}$ It is assumed that the growth of platinum oxide was restrained at the surface of platinum covered by a hydrogel. A low localized $\mathrm{pH}$ in the hydrogel is possible because of (a) the presence of protons trapped in the hydrogels during anodic gelation, (b) faster diffusion of protons than of water molecules, and (c) negatively charged cellulose facilitating trapping of protons into the hydrogel.

The TOCN hydrogel (without Triton X-100) was found to be less stable during these measurements, as the hydrogel was destroyed at the increased rotation rate of $2000 \mathrm{rpm}$ or at higher current density $\left(-10 \mathrm{~mA} / \mathrm{cm}^{2}\right)$. On the contrary, the Triton X100 containing TOCN gel still showed the good performance as an ORR preventing protective layer under these conditions (Figure 9B,C). The TOCN Triton X-100 hydrogels blocked $80 \%$ of ORR reaction at $2000 \mathrm{rpm}$ (at potential $-0.74 \mathrm{~V}$ ), and still $75 \%$ even after galvanostatic HER. The stability of the hydrogels was further evaluated in galvanostatic HER measurements (Figure 9C). The CV results (purple line in Figure 9B) show that the TOCN-Triton X-100 gel stayed on the surface of the Pt-RDE, even after galvanostatic HER, preventing ORR without being destroyed. We believe that the Triton X-100 contributes to improved plasticity of the hydrogel, with higher tolerance against shear forces and transport of hydrogen.

There was no difference in ECSA of a Pt electrode in the presence/absence of the TOCN hydrogel surface coating (Figure 10A), indicating that the hydrogel is physically, and not chemically, bound on the electrode surface. Nonetheless, the hydrogel can suppress the hydrogen oxidation reaction (HOR) as well as ferricyanide reduction. During the hydrogen oxidation (Figure 10B), the limiting current density of bare Pt was $\sim 0.167$ $\mathrm{mA} / \mathrm{cm}^{2}$, while on the gel coated Pt electrodes it was lower than $0.03 \mathrm{~mA} / \mathrm{cm}^{2}$, which means that the HOR was suppressed by $82 \%$. The limiting current density of ferricyanide reduction (Figure 10C) of bare Pt was $j \approx-4.4 \mathrm{~mA} / \mathrm{cm}^{2}$, which is 3 time higher than those of the gel coated $\mathrm{Pt}, j \approx-1.5 \mathrm{~mA} / \mathrm{cm}^{2}(67 \%$ suppressed). It is assumed that hydrogel restricts the accessibility of molecules from bulk electrolyte toward electrode, working as a barrier.

\section{CONCLUSION}

$\mathrm{pH}$ responsive TOCN hydrogels can be electrochemically precipitated on the surface of conductive materials by applying a certain anodic current for a relatively short duration ( $<1 \mathrm{~min})$. A local $\mathrm{pH}$ decrease, induced by electrochemical water oxidation, leads to protonation of TOCN, which neutralizes the surface charge and triggers a sol-gel transition of TOCN to hydrogel and hence its precipitation on the anode substrate. Not only TOCN but also nano- or micrometer sized particles containing hydrogel composites can be electrochemically immobilized on conductive surfaces from simple aqueous dispersions of these particles and TOCN. We demonstrate the possibility of TOCN as a coating agent for multiple layers of hydrogels or composite 
(A)

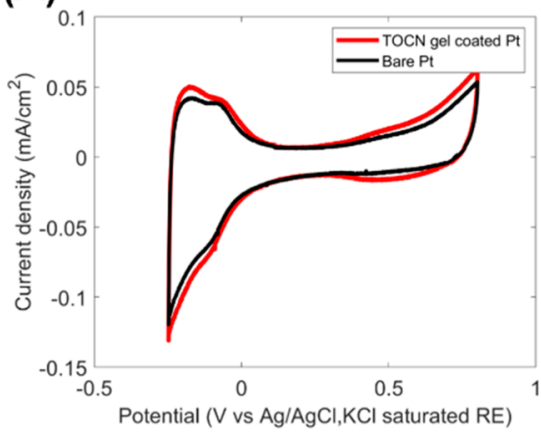

(B)

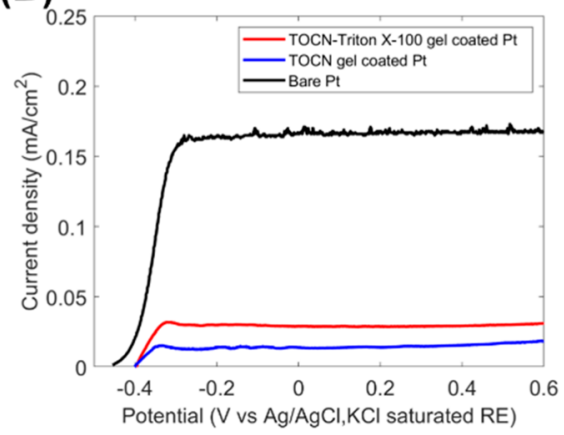

(C)

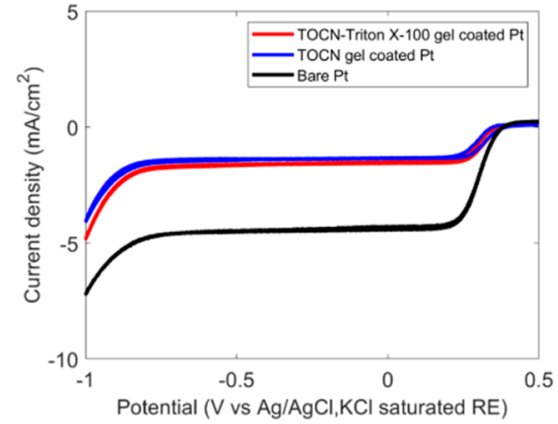

Figure 10. Electrochemical measurements of performance of Pt electrode in the presence/absence of the TOCN hydrogel surface coating in the range of (A) hydrogen adsorption evaluating ECSA, (B) hydrogen oxidation reaction, and $(\mathrm{C}) \mathrm{K}_{3} \mathrm{Fe}(\mathrm{CN})_{6}$ reduction reaction.

materials for versatile applications, such as lithium ion battery electrode. The TOCN hydrogel itself can work as an ORR protective layer during HER, through which water, hydroxide ions, and hydrogen can penetrate, while it limits the transport of oxygen. By adding a surfactant to the deposition solution, the stability of the hydrogel as well as its performance as ORR protective layer can be further improved. As it is a facile coating technique, and performed in unharmful aqueous suspensions leading to the formation of different functional coatings, we believe that this technique might find its application in diverse fields ranging from biomedical engineering to energy devices.

\section{ASSOCIATED CONTENT}

\section{S Supporting Information}

The Supporting Information is available free of charge at https://pubs.acs.org/doi/10.1021/acssuschemeng.9b04171.

Photographs of the hydrogel by $\mathrm{pH}$-induced sol-gel transition, TGA, graphite/TOCN hydrogel deposition mass loading varying deposition conditions, microscopic image of lithium iron phosphate particles, $\mathrm{CV}$ and galvanostatic HER (PDF)

Video 1: $\mathrm{pH}$ profile of the coating medium during watersplitting induced electro-precipitation of TOCN hydrogel on the surface of a carbon paper (MP4)

Video 2: $\mathrm{pH}$ profile of the coating medium during watersplitting induced electro-precipitation of TOCN hydrogel on the surface of a copper foil (MP4)

\section{AUTHOR INFORMATION}

\section{Corresponding Author}

*E-mail: hyeyun@kth.se.

\section{ORCID}

Hyeyun Kim: 0000-0001-9404-4087

Balázs Endródi: 0000-0003-3237-9222

Ann Cornell: 0000-0001-5816-2924

\section{Notes}

The authors declare no competing financial interest.

\section{ACKNOWLEDGMENTS}

This work was supported by the Knut and Alice Wallenberg Foundation, and the authors also thank the Wallenberg Wood Science Center for all financial and technical support.

\section{REFERENCES}

(1) Geng, Z.; Wang, X.; Guo, X.; Zhang, Z.; Chen, Y.; Wang, Y. Electrodeposition of Chitosan Based on Coordination with Metal Ions in Situ-Generated by Electrochemical Oxidation. J. Mater. Chem. B 2016, 4 (19), 3331-3338.

(2) Suginta, W.; Khunkaewla, P.; Schulte, A. Electrochemical Biosensor Applications of Polysaccharides Chitin and Chitosan. Chem. Rev. 2013, 113 (7), 5458-5479.

(3) Cheng, Y.; Luo, X.; Betz, J.; Payne, G. F.; Bentley, W. E.; Rubloff, G. W. Mechanism of Anodic Electrodeposition of Calcium Alginate. Soft Matter 2011, 7 (12), 5677-5684.

(4) Gray, K. M.; Liba, B. D.; Wang, Y.; Cheng, Y.; Rubloff, G. W.; Bentley, W. E.; Montembault, A.; Royaud, I.; David, L.; Payne, G. F. Electrodeposition of a Biopolymeric Hydrogel: Potential for One-Step Protein Electroaddressing. Biomacromolecules 2012, 13 (4), 11811189.

(5) Cheong, M.; Zhitomirsky, I. Electrodeposition of Alginic Acid and Composite Films. Colloids Surf., A 2008, 328 (1-3), 73-78.

(6) Wang, Z.; Zhang, X.; Gu, J.; Yang, H.; Nie, J.; Ma, G. Electrodeposition of Alginate/Chitosan Layer-by-Layer Composite Coatings on Titanium Substrates. Carbohydr. Polym. 2014, 103 (1), $38-45$.

(7) Taira, N.; Ino, K.; Kunikata, R.; Shiku, H.; Matsue, T.; Terauchi, M.; Gakumasawa, M.; Suda, A. Local Hydrogel Fabrication Based on Electrodeposition with a Large-Scale Integration (LSI)-Based Amperometric Device. Sens. Actuators, B 2018, 277, 95-101.

(8) Cheng, Y.; Luo, X.; Betz, J.; Buckhout-White, S.; Bekdash, O.; Payne, G. F.; Bentley, W. E.; Rubloff, G. W. In Situ Quantitative Visualization and Characterization of Chitosan Electrodeposition with Paired Sidewall Electrodes. Soft Matter 2010, 6 (14), 3177-3183.

(9) De France, K. J.; Hoare, T.; Cranston, E. D. Review of Hydrogels and Aerogels Containing Nanocellulose. Chem. Mater. 2017, 29 (11), 4609-4631.

(10) Way, A. E.; Hsu, L.; Shanmuganathan, K.; Weder, C.; Rowan, S.J. PH-Responsive Cellulose Nanocrystal Gels and Nanocomposites. ACS Macro Lett. 2012, 1 (8), 1001-1006.

(11) Wang, Y.; Zhang, Z.; Wang, M.; Guo, C.; Liu, H.; Zeng, H.; Duan, X.; Zhou, Y.; Tang, Z. Direct Electrodeposition of Carboxymethyl Cellulose Based on Coordination Deposition Method. Cellulose 2018, 25 (1), 105-115.

(12) Rizwan, M.; Yahya, R.; Hassan, A.; Yar, M.; Azzahari, A. D.; Selvanathan, V.; Sonsudin, F.; Abouloula, C. N. PH Sensitive Hydrogels in Drug Delivery: Brief History, Properties, Swelling, and Release Mechanism, Material Selection and Applications. Polymers 2017, 9 (12), 137.

(13) Fall, A. B.; Lindström, S. B.; Sundman, O.; Ödberg, L.; Wå gberg, L. Colloidal Stability of Aqueous Nanofibrillated Cellulose Dispersions. Langmuir 2011, 27 (18), 11332-11338.

(14) Mittal, N.; Ansari, F.; Gowda Krishne, V.; Brouzet, C.; Chen, P.; Larsson, P. T.; Roth, S. V.; Lundell, F.; Wågberg, L.; Kotov, N. A.; Söderberg, L. D. Multiscale Control of Nanocellulose Assembly: Transferring Remarkable Nanoscale Fibril Mechanics to Macroscale Fibers. ACS Nano 2018, 12 (7), 6378-6388.

(15) Fujisawa, S.; Okita, Y.; Fukuzumi, H.; Saito, T.; Isogai, A. Preparation and Characterization of TEMPO-Oxidized Cellulose 
Nanofibril Films with Free Carboxyl Groups. Carbohydr. Polym. 2011, 84 (1), 579-583.

(16) Sato, Y.; Kusaka, Y.; Kobayashi, M. Charging and Aggregation Behavior of Cellulose Nanofiber in Aqueous Solution. Langmuir 2017, 33, 12660-12669.

(17) Wu, L. Q.; Lee, K.; Wang, X.; English, D. S.; Losert, W.; Payne, G. F. Chitosan-Mediated and Spatially Selective Electrodeposition of Nanoscale Particles. Langmuir 2005, 21 (8), 3641-3646.

(18) Lu, H.; Guccini, V.; Kim, H.; Salazar-Alvarez, G.; Lindbergh, G.; Cornell, A. Effects of Different Manufacturing Processes on TEMPOOxidized Carboxylated Cellulose Nanofiber Performance as Binder for Flexible Lithium-Ion Batteries. ACS Appl. Mater. Interfaces 2017, 9 (43), 37712-37720.

(19) Saito, T.; Kimura, S.; Nishiyama, Y.; Isogai, A. Cellulose Nanofibers Prepared by TEMPO-Mediated Oxidation of Native Cellulose. Biomacromolecules 2007, 8 (8), 2485-2491.

(20) Katz, S.; Beatson, R. P.; M, S. A. SCAN-CM 65:02 Total Acidic Group Content. Scand. Pulp, Pap. Board Test. Comm. 2002, 1-4.

(21) Schneider, C. A.; Rasband, W. S.; Eliceiri, K. W. NIH Image to ImageJ: 25 Years of Image Analysis. Nat. Methods 2012, 9 (7), 671675.

(22) Zamburlini, E.; Jensen, K. D.; Stephens, I. E. L.; Chorkendorff, I.; Escudero-Escribano, M. Benchmarking Pt and Pt-Lanthanide Sputtered Thin Films for Oxygen Electroreduction: Fabrication and Rotating Disk Electrode Measurements. Electrochim. Acta 2017, 247, $708-721$.

(23) Mayrhofer, K. J. J.; Strmcnik, D.; Blizanac, B. B.; Stamenkovic, V.; Arenz, M.; Markovic, N. M. Measurement of Oxygen Reduction Activities via the Rotating Disc Electrode Method: From Pt Model Surfaces to Carbon-Supported High Surface Area Catalysts. Electrochim. Acta 2008, 53 (7), 3181-3188.

(24) Isobe, N.; Chen, X.; Kim, U. J.; Kimura, S.; Wada, M.; Saito, T.; Isogai, A. TEMPO-Oxidized Cellulose Hydrogel as a High-Capacity and Reusable Heavy Metal Ion Adsorbent. J. Hazard. Mater. 2013, 260, 195-201.

(25) Chen, R.; Yang, C.; Cai, W.; Wang, H. Y.; Miao, J.; Zhang, L.; Chen, S.; Liu, B. Use of Platinum as the Counter Electrode to Study the Activity of Nonprecious Metal Catalysts for the Hydrogen Evolution Reaction. ACS Energy Lett. 2017, 2 (5), 1070-1075.

(26) Leijonmarck, S.; Cornell, A.; Lindbergh, G.; Wågberg, L. Flexible Nano-Paper-Based Positive Electrodes for Li-Ion Batteries-Preparation Process and Properties. Nano Energy 2013, 2 (5), 794-800.

(27) Lu, H.; Behm, M.; Leijonmarck, S.; Lindbergh, G.; Cornell, A. Flexible Paper Electrodes for Li-Ion Batteries Using Low Amount of TEMPO-Oxidized Cellulose Nanofibrils as Binder. ACS Appl. Mater. Interfaces 2016, 8 (28), 18097-18106.

(28) Lalau, C. C.; Low, C. T. J. Electrophoretic Deposition for Lithium-Ion Battery Electrode Manufacture. Batter. Supercaps 2019, 2 (6), 551-559.

(29) Van der Biest, O.; Vandeperre, L. Electrophoretic Deposition of Materials. Annu. Rev. Mater. Sci. 1999, 29 (1), 327-352.

(30) Boccaccini, A. R.; Cho, J.; Roether, J. A.; Thomas, B. J. C.; Jane Minay, E.; Shaffer, M. S. P. Electrophoretic Deposition of Carbon Nanotubes. Carbon 2006, 44 (15), 3149-3160.

(31) Seo, S. D.; Hwang, I. S.; Lee, S. H.; Shim, H. W.; Kim, D. W. 1D/ 2D Carbon Nanotube/Graphene Nanosheet Composite Anodes Fabricated Using Electrophoretic Assembly. Ceram. Int. 2012, 38 (4), 3017-3021.

(32) Schweidler, S.; De Biasi, L.; Schiele, A.; Hartmann, P.; Brezesinski, T.; Janek, J. Volume Changes of Graphite Anodes Revisited: A Combined Operando X-Ray Diffraction and in Situ Pressure Analysis Study. J. Phys. Chem. C 2018, 122 (16), 8829-8835.

(33) Dahn, J. R. Phase Diagram of LixC6. Phys. Rev. B: Condens. Matter Mater. Phys. 1991, 44 (17), 9170-9177.

(34) Crabtree, G. W.; Dresselhaus, M. S.; Buchanan, M. V. The Hydrogen Economy. Phys. Today 2004, 57 (12), 39-44.

(35) Qureshi, M.; Shinagawa, T.; Tsiapis, N.; Takanabe, K. Exclusive Hydrogen Generation by Electrocatalysts Coated with an Amorphous
Chromium-Based Layer Achieving Efficient Overall Water Splitting. ACS Sustainable Chem. Eng. 2017, 5 (9), 8079-8088.

(36) Maeda, K.; Teramura, K.; Lu, D.; Saito, N.; Inoue, Y.; Domen, K. Roles of $\mathrm{Rh} / \mathrm{Cr} 2 \mathrm{O} 3$ (Core/Shell) Nanoparticles Photodeposited on Visible-Light-Responsive (Ga1-XZn x)(N1-XOx) Solid Solutions in Photocatalytic Overall Water Splitting. J. Phys. Chem. C 2007, 111 (20), $7554-7560$

(37) Maeda, K.; Teramura, K.; Lu, D.; Saito, N.; Inoue, Y.; Domen, K. Noble-Metal/Cr2O3 Core/Shell Nanoparticles as a Cocatalyst for Photocatalytic Overall Water Splitting. Angew. Chem., Int. Ed. 2006, 45 (46), 7806-7809.

(38) Eisenberg, D.; Ahn, H. S.; Bard, A. J. Enhanced Photoelectrochemical Water Oxidation on Bismuth Vanadate by Electrodeposition of Amorphous Titanium Dioxide. J. Am. Chem. Soc. 2014, 136 (40), 14011-14014

(39) Matsumoto, Y.; Unal, U.; Tanaka, N.; Kudo, A.; Kato, H. Electrochemical Approach to Evaluate the Mechanism of Photocatalytic Water Splitting on Oxide Photocatalysts. J. Solid State Chem. 2004, 177 (11), 4205-4212.

(40) Han, K.; Kreuger, T.; Mei, B.; Mul, G. Transient Behavior of Ni@ NiOx Functionalized SrTiO3 in Overall Water Splitting. ACS Catal. 2017, 7 (3), 1610-1614.

(41) Yuan, G.; Agiral, A.; Pellet, N.; Kim, W.; Frei, H. Inorganic CoreShell Assemblies for Closing the Artificial Photosynthetic Cycle. Faraday Discuss. 2014, 176, 233-249.

(42) Bau, J. A.; Takanabe, K. Ultrathin Microporous SiO2Membranes Photodeposited on Hydrogen Evolving Catalysts Enabling Overall Water Splitting. ACS Catal. 2017, 7 (11), 7931-7940.

(43) Labrador, N. Y.; Songcuan, E. L.; De Silva, C.; Chen, H.; Kurdziel, S. J.; Ramachandran, R. K.; Detavernier, C.; Esposito, D. V. Hydrogen Evolution at the Buried Interface between Pt Thin Films and Silicon Oxide Nanomembranes. ACS Catal. 2018, 8 (3), 1767-1778.

(44) Endrődi, B.; Smulders, V.; Simic, N.; Wildlock, M.; Mul, G.; Mei, B.; Cornell, A. In Situ Formed Vanadium-Oxide Cathode Coatings for Selective Hydrogen Production. Appl. Catal., B 2019, 244, 233-239.

(45) Masuda, T.; Fukumitsu, H.; Fugane, K.; Togasaki, H.; Matsumura, D.; Tamura, K.; Nishihata, Y.; Yoshikawa, H.; Kobayashi, K.; Mori, T.; Uosaki, K.; et al. Role of Cerium Oxide in the Enhancement of Activity for the Oxygen Reduction Reaction at Pt$\mathrm{CeO}$ x Nanocomposite Electrocatalyst - An in Situ Electrochemical XRay Absorption Fine Structure Study. J. Phys. Chem. C 2012, 116 (18), 10098-10102. 